University of Wollongong

Research Online

Faculty of Engineering - Papers (Archive)

Faculty of Engineering and Information

Sciences

$1-1-2007$

\title{
Nonlinear optical properties of semiconductor quantum wells under intense terahertz radiation
}

T. Y. Zhang

Chinese Academy of Sciences

W. Zhao

Chinese Academy of Sciences

X. M. Liu

Chinese Academy of Sciences

C. Zhang

University of Wollongong, czhang@uow.edu.au

Follow this and additional works at: https://ro.uow.edu.au/engpapers

Part of the Engineering Commons

https://ro.uow.edu.au/engpapers/3277

\section{Recommended Citation}

Zhang, T. Y.; Zhao, W.; Liu, X. M.; and Zhang, C.: Nonlinear optical properties of semiconductor quantum wells under intense terahertz radiation 2007, 041909-1-041909-3.

https://ro.uow.edu.au/engpapers/3277

Research Online is the open access institutional repository for the University of Wollongong. For further information contact the UOW Library: research-pubs@uow.edu.au 


\title{
Nonlinear optical properties of semiconductor quantum wells under intense terahertz radiation
}

\author{
T. Y. Zhang, ${ }^{\text {a) }}$ W. Zhao, and X. M. Liu \\ State Key Laboratory of Transient Optics and Photonics, Xi'an Institute of Optics and Precision Mechanics, \\ Chinese Academy of Sciences, 17 Xinxi Road, Xi'an 710119, People's Republic of China \\ C. Zhang \\ School of Engineering Physics, University of Wollongong, New South Wales 2522, Australia
}

(Received 13 June 2007; accepted 26 June 2007; published online 26 July 2007)

\begin{abstract}
The nonlinear optical properties of semiconductor quantum wells driven by intense in-plane terahertz electric fields are investigated theoretically by employing the extended semiconductor Bloch equations. The dynamical Franz-Keldysh effect of the optical absorption near the band edge is analyzed with Coulomb correlation among the carriers included. The in-plane terahertz field induces a variety of behavior in the absorption spectra, including terahertz replicas of the (dark) $2 p$ exciton and terahertz sidebands of the $1 s$ exciton. The dependence of these interesting features on the intensity, frequency, and phase of the terahertz field is explored in detail. (C) 2007 American Institute of Physics. [DOI: 10.1063/1.2760040]
\end{abstract}

The effect of an applied electric field on the optical properties of semiconductor structures plays an importance in understanding many interesting phenomena in semiconductors. An applied static electric field leads to the well-known Franz-Keldysh effect (FKE), i.e., pronounced oscillations occurring in the optical absorption spectrum above the band gap and nonvanishing exponential absorption tail below the band gap. ${ }^{1,2}$ Recently, the FKE is extended to nonlinear twophoton and multiphoton absorption. ${ }^{3}$ The FKE is also extended to time-dependent electric fields, i.e., the dynamical Franz-Keldysh effect (DFKE). The DFKE predicts that the band edge should blueshift by the ponderomotive energy, $E_{\text {pon }}=e^{2} F_{0}^{2} / 4 m \Omega^{2}$ (i.e., the average kinetic energy of a particle of mass $m$ and charge $e$ in an electric field $F_{0} \cos \Omega t$ ), thus inducing the above-band-gap transparency and increasing the below gap absorption. ${ }^{4-7}$ Since the optical absorption spectrum near the band gap is dominated by exciton effects, which manifest as strong resonances occurring at bound exciton states and enhanced continuum absorption, the DFKE is subsequently extended to include the exciton effects, which manifest as a blueshift of the exciton peak. ${ }^{8,9}$ Theoretical work on the DFKE has been based on various formulations, such as Green's function techniques, ${ }^{6-8,10}$ the extended semiconductor Bloch equations (SBE's) to include the terahertz ac field, ${ }^{11,12}$ and the inhomogenous Schrodinger equation in real space to show the distribution of the resulted anisotropic and distorted wave packet. ${ }^{13,14}$ In this letter, we calculate the optical absorption of an ultrashort interband probe pulse in a two-dimensional (2D) semiconductor pumped by a cw-terahertz beam. The influence of the terahertz pump beam with various parameters on the absorption of the probe beam is explored. Though similar configurations have been studied before, the calculations based on Green's function techniques ${ }^{8,9}$ invoked Floquet state expansions or inverted a large matrix that resulted from integral equations, thus needing huge computational effort. In addition, the dependence of absorption on the phase of the terahertz field was missed. Furthermore, investigations presented before mostly focused on features above the main exciton

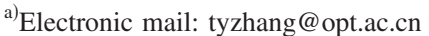

resonance. ${ }^{11,12}$ However, we show that the optical absorption below the band edge is highly sensitive to the phase of the terahertz field, and the sidebands below the main exciton peak are more prominent than those above the main exciton peak. By controlling the arrival time of the ultrashort optical pulse at different phases of the terahertz field, we can change the optical response from absorption to gain.

The absorption coefficient $\alpha(\omega)$ of a dielectric medium is related to the optical dielectric function $\epsilon(\omega)$ by

$$
\alpha(\omega)=\frac{\omega}{n(\omega) c} \epsilon^{\prime \prime}(\omega),
$$

where $n(\omega)=\sqrt{\frac{1}{2}\left[\epsilon^{\prime}(\omega)+\sqrt{\epsilon^{\prime 2}(\omega)+\epsilon^{\prime \prime 2}(\omega)}\right]}$ is the index of refraction, $c$ is the speed of light in free space, and $\epsilon^{\prime}(\omega)$ and $\epsilon^{\prime \prime}(\omega)$ are the real and imaginary parts of $\epsilon(\omega)$, respectively. The optical dielectric function is related to the optical susceptibility $\chi(\omega)$ by $\epsilon(\omega)=1+\chi(\omega)$. While the optical susceptibility relates the excitation optical field $\mathcal{E}(t)$ with its induced macroscopic polarization $P(t)$ via

$$
P(t)=\epsilon_{0} \int_{-\infty}^{t} d t^{\prime} \chi\left(t ; t^{\prime}\right) \mathcal{E}\left(t^{\prime}\right),
$$

where $\epsilon_{0}$ is the vacuum permittivity. By performing a Fourier transform of Eq. (2), $\chi(\omega)$ can be obtained as

$$
\chi(\omega) \equiv \frac{\widetilde{P}(\omega)}{\epsilon_{0} \widetilde{\mathcal{E}}(\omega)},
$$

where $\widetilde{\mathcal{E}}(\omega)$ and $\widetilde{P}(\omega)$ are the Fourier transform of the optical field $\mathcal{E}(t)$ and the polarization function $P(t)$. The macroscopic interband polarization $P(t)$ is the sum of the microscopic interband polarization, $P(t)=2 d_{c v} \Sigma_{\mathbf{k}} p_{\mathbf{k}}(t)$, where $d_{c v}$ is the interband dipole matrix element and the factor 2 is due to the spin degeneracy. In the two-band model, the microscopic interband polarization is defined as $p_{\mathbf{k}}(t)=\left\langle\beta_{-\mathbf{k}} \alpha_{\mathbf{k}}\right\rangle$, where $\alpha_{\mathbf{k}}\left(\beta_{-\mathbf{k}}\right)$ is the annihilation operator of an electron (a hole) with wavevector $\mathbf{k}(-\mathbf{k})$ in the conduction (valence) band. Under the Hartree-Fock approximation, from the Heisenberg equations of motion for the electron and hole 
operators, we can derive the well-known SBE's for microscopic polarization $p_{\mathbf{k}}(t)$ and carrier populations $n_{\mathbf{k}}^{e}=\left\langle\alpha_{\mathbf{k}}^{\dagger} \alpha_{\mathbf{k}}\right\rangle$ and $n_{\mathbf{k}}^{h}=\left\langle\beta_{-\mathbf{k}}^{\dagger} \beta_{-\mathbf{k}}\right\rangle$ as ${ }^{11,12,15-18}$

$$
\begin{aligned}
\frac{\partial}{\partial t} p_{\mathbf{k}}= & -\frac{i}{\hbar}\left(e_{\mathbf{k}}^{e}+e_{\mathbf{k}}^{h}\right) p_{\mathbf{k}}-i\left(n_{\mathbf{k}}^{e}+n_{\mathbf{k}}^{h}-1\right) \Omega_{R, \mathbf{k}}-\frac{e}{\hbar} \mathbf{F} \cdot \nabla_{\mathbf{k}} p_{\mathbf{k}} \\
& +\left.\frac{\partial}{\partial t} p_{\mathbf{k}}\right|_{\text {scatt }}, \\
\frac{\partial}{\partial t} n_{\mathbf{k}}^{e}= & -2 \operatorname{Im}\left[\Omega_{R, \mathbf{k}} p_{\mathbf{k}}^{*}\right]-\frac{e}{\hbar} \mathbf{F} \cdot \nabla_{\mathbf{k}} n_{\mathbf{k}}^{e}+\left.\frac{\partial}{\partial t} n_{\mathbf{k}}^{e}\right|_{\text {scatt }}, \\
\frac{\partial}{\partial t} n_{\mathbf{k}}^{h}= & -2 \operatorname{Im}\left[\Omega_{R, \mathbf{k}} p_{\mathbf{k}}^{*}\right]-\frac{e}{\hbar} \mathbf{F} \cdot \nabla_{\mathbf{k}} n_{\mathbf{k}}^{h}+\left.\frac{\partial}{\partial t} n_{\mathbf{k}}^{h}\right|_{\text {scatt }},
\end{aligned}
$$

where $e_{\mathbf{k}}^{\lambda}=\left(E_{\mathbf{k}}^{\lambda}-\Sigma_{\mathbf{q}} V_{|\mathbf{k}-\mathbf{q}|} n_{\mathbf{q}}^{\lambda}\right) / \hbar$ are the electron and hole energies renormalized by Coulomb interactions, $E_{\mathbf{k}}^{(e, h)}$ are the electron and hole band dispersions, $\Omega_{R, \mathbf{k}}$ $=\left(d_{c v} \mathcal{E}(t)+\Sigma_{\mathbf{q} \neq \mathbf{k}} V_{|\mathbf{k}-\mathbf{q}|} P_{\mathbf{q}}\right) / \hbar$ is the generalized Rabi frequency, and $V_{\mathbf{q}}=\left(e^{2} / 2 \epsilon_{0} \epsilon\right)(1 / \mathbf{q})$ is the Fourier transform of ideal 2D Coulomb potential. The effect of the finite width of a realistic quantum well can be accounted for by introducing a form factor into the Coulomb potential. The scattering terms in Eq. (4) represent the higher-order Coulomb correlations between carriers and carrier-phonon collisions; $\mathbf{F}_{\mathrm{THz}}(t)=\mathbf{F}_{0} \cos (\Omega t-\theta)$ is the in-plane driving terahertz field, with $\mathbf{F}_{0}, \Omega, \theta$ as the amplitude, frequency, and phase, respectively.

Without the applied in-plane terahertz field, the SBE's have a cylindrical symmetry in the $2 \mathrm{D}$ case. The applied terahertz field breaks the cylindrical symmetry. It is thus necessary to solve the extended SBE's on a 2D grid of points in $\mathbf{k}$ space. The calculation on this $2 \mathrm{D}$ grid is very time consuming. Therefore, we introduce a moving frame of reference $\mathbf{k}^{\prime}=\mathbf{k}+e / \hbar \int^{t} \mathbf{F}_{\mathrm{THz}}\left(t^{\prime}\right) d t^{\prime}$ for the numerical convenience. The transformed SBE's are then formally the same as the field-free SBE's, except that the quasimomentum obeys the so-called acceleration theorem $(\partial / \partial t) \mathbf{k}^{\prime}=(e / \hbar) \mathbf{F}_{\mathrm{THz}}(t)$; thus, the band dispersions oscillate in $\mathbf{k}$ space.

In our numerical calculation, a purely 2D Coulomb potential and typical GaAs parameters were used. We make the relaxation-time approximation for the polarization dephasing with the dephasing time of 2 ps. The envelope of the interband probe pulse is assumed to be Gaussian and centered at time $t=10 \tau$, with a full width at half maximum of $\tau 50 \mathrm{fs}$, and the central frequency is in resonance with the bare band edge. Since it is difficult to lock the arrival of the probe optical pulse to a particular phase of the terahertz field at present, we make phase-averaged theoretical calculations of the absorption in order to compare theory with experimental results.

Figures 1(a) and 1(b) show the intensity dependence of the optical absorption spectrum of a 2D semiconductor driven by an in-plane terahertz field with frequencies of $2.5 \mathrm{THz}(10.3 \mathrm{meV})$ and $3.5 \mathrm{THz}(14.42 \mathrm{meV})$, respectively. The two selected frequencies are near resonant with the $1 s-2 p$ transition but are tuned below or above the $1 s-2 p$ transition. Compared to the field-free absorption, the terahertz field induces interesting features in the spectrum. One remarkable feature is that the peak appeared in the vicinity of the $1 s$ peak. This induced peak is $1 \mathrm{THz}$ photon energy below the $2 s$ peaks. Essentially, it is the $2 p$ replica due to the
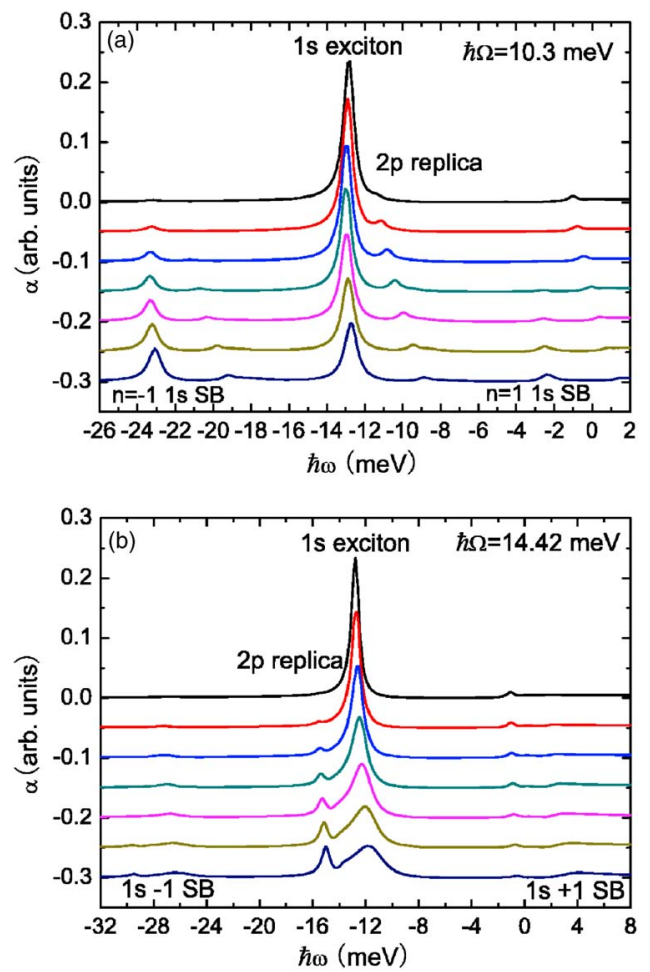

FIG. 1. (Color online) Absorption spectra for terahertz frequencies of $2.5 \mathrm{THz}(10.3 \mathrm{meV})$ (a) and $3.5 \mathrm{THz}(14.42 \mathrm{meV})$ (b). In each case, the amplitudes of the terahertz fields are (from top to bottom) $1.0, \ldots$, $7.0 \mathrm{kV} / \mathrm{cm}$.

possibility of creating a $2 p$ exciton (degenerate with the $2 s$ exciton) by simultaneous absorption of one probe photon and $1 \mathrm{THz}$ photon; i.e., the terahertz field gives rise to the appearance of otherwise dark exciton states. This was first demonstrated using Green's function methods. ${ }^{8}$ The terahertz field also leads to shifts of the main features. A detailed analysis revealed that the $1 \mathrm{~s}$ exciton peak shifts in different ways for the two selected frequencies as the intensity increases. For the frequency of $10.3 \mathrm{meV}$, the $1 \mathrm{~s}$ peak redshifts and reaches a maximum and reverses, becoming blueshifts. While for the frequency of $14.42 \mathrm{meV}$, the $1 \mathrm{~s}$ peak blueshifts for all terahertz intensities. Since the effect of the DFKE itself is to blueshift all the main features at all terahertz intensities, the redshift of the $1 s$ exciton peak for $10.3 \mathrm{meV}$ can only be understood by considering another effect of the terahertz field, the ac Stark effect. The direction of the ac Stark shifts depends on whether the terahertz field is tuned above or below the $1 s-2 p$ transition. The interplay between the DFKE and ac Stark effect determines the direction and size of the shifts in the $1 s$ peak and the $2 p$ replica. According to the ac Stark effect, if the driving frequency is tuned below the $1 s-2 p$ resonant transition, the two levels will repel with respect to the external probe, while if the driving frequency is tuned above the $1 s-2 p$ resonant transition, the two levels will attract; if the frequency is tuned to the resonant transition, an apparent splitting of the resonances will occur (Autler-Townes splitting). ${ }^{19-21}$ So, for a frequency of $14.42 \mathrm{meV}$, the ac Stark effect blueshifts the $1 s$ state and redshifts the $2 p$ state, and for a frequency of $10.3 \mathrm{meV}$, the Stark shifts are in the opposite directions. Therefore, at higher intensities the DFKE dominates and a net blueshift occurs. Apart from the shifts, the $1 s$ peak broadens as the field strength increases. This is presumably due to the terahertz-induced oscillations in the relative position of the 


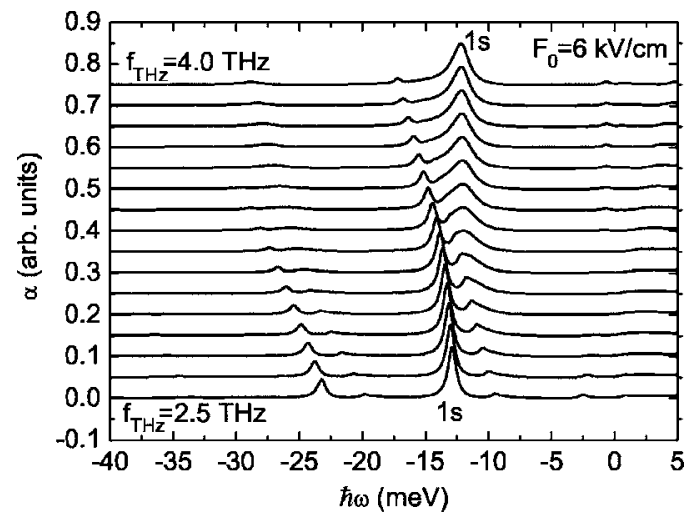

FIG. 2. Absorption spectra for terahertz frequencies (from bottom to top) $2.5,2.6, \ldots, 4.0 \mathrm{THz}$. The $2 p$ replica anticrosses the $1 s$ exciton peak when the terahertz frequency increases from below to above the $1 s-2 p$ transition energy.

bound electron and hole-when these are comparable in size with the exciton, we expect the corresponding absorption feature to be significantly broadened. Another interesting feature is the appearance of the $1 s$ sideband. The $1 s$ sideband corresponds to the absorption of a single interband photon, together with the emission or absorption of terahertz photons depending on the sideband index. ${ }^{22,23}$

Figure 2 depicts the frequency dependence of the optical absorption spectrum of a $2 \mathrm{D}$ semiconductor driven by an in-plane terahertz field with a field strength of $6 \mathrm{kV} / \mathrm{cm}$. As the frequency of the terahertz field increases from 2.5 to $4.0 \mathrm{THz}$, the $2 p$ replica anticrosses with the $1 s$ exciton peak from $3 \mathrm{meV}$ at the higher energy side to $5 \mathrm{meV}$ at the lower energy side of the $1 s$ exciton peak. The main $1 s$ exciton peak broadens when the frequency is tuned above the $1 s-2 p$ transition. We attribute this broadening to the breaking up of the $1 s$ exciton as the terahertz photon energy is approximately equivalent to the $1 s$ exciton binding energy. The \pm 1 sidebands of the main $1 s$ exciton are also evident but are asymmetric.

As mentioned above, presently, it is difficult to lock the arrival of the probe pulse to a particular phase. However, the investigation of the phase dependence of the absorption should shed light on the fundamental physics of the carrierphoton interaction in quantum wells. Our results also indicate that if the phase locking difficulty can be overcome in the future, we will have the capability to control the transition from absorption to gain. This may lead to a wider application of quantum wells as optical switches or modulators. The dependence of the nonlinear optical features on the phase of the terahertz field at the time of the optical pulse arrival is shown in Fig. 3. Varying the phase of the terahertz field causes a significant change in the shape of the sideband absorption characteristics. The characteristics evolve from absorption peaks to gain peaks. The steep transition from absorption to gain occurs at the position of the sideband. Remarkably, the sidebands below the main exciton peak are much more pronounced than those above the main exciton peak. In contrast, the $2 p$ replica have a very weak phase dependence.

In conclusion, we presented a detailed study of the effects of an in-plane terahertz electric field on the absorption spectrum of GaAs quantum wells. The dependence of the optical properties on the intensity, frequency, and phase of

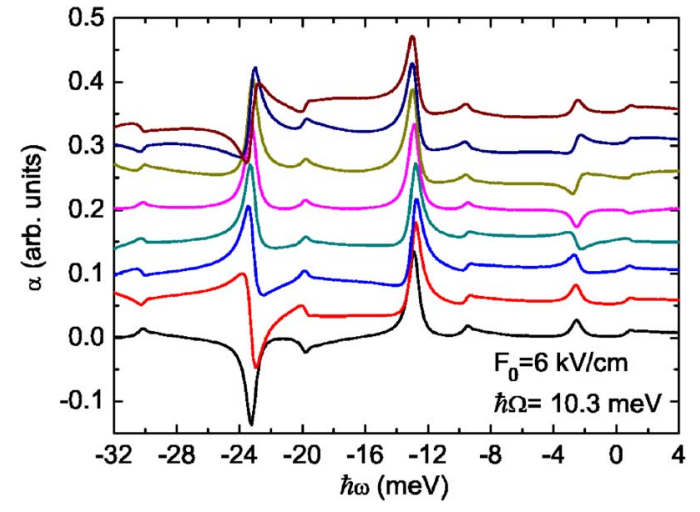

FIG. 3. (Color online) Absorption spectra for amplitudes of the terahertz fields of $6.0 \mathrm{kV} / \mathrm{cm}$ and terahertz frequency of $2.5 \mathrm{THz}$. From bottom to top, the phases of the terahertz field are $0 \pi / 4, \pi / 4, \ldots, 7 \pi / 4$.

the terahertz field is analyzed and discussed. Near the band edge, there are two principal terahertz generated features. One is the appearance of the $2 p$ replica peak in the gap. The other is the $1 s$ sideband resonances appearing above and below the main $1 s$ peak. The sidebands below and above the main exciton peak are asymmetric, and the sidebands below the main exciton peak are more remarkable than those above the main exciton peak. The sidebands are highly sensitive to the phase of the terahertz field, while the main $1 s$ exciton peak and the single-photon terahertz replica of the $2 p$ peak depend very weakly on the phase of the terahertz field.

This work is supported by the National Natural Science Foundation of China (10390160, 10604066), National Basic Research Program of China (973 Program) (2007CB310405), the China Postdoctoral Science Foundation, and K. C. Wong Education Foundation, Hong Kong.

${ }^{1}$ W. Z. Franz, Z. Naturforsch. A 13a, 484 (1958).

${ }^{2}$ L. V. Keldysh, Zh. Eksp. Teor. Fiz. 34, 1118 (1958) [Sov. Phys. JETP 7, 788 (1958)].

${ }^{3}$ H. Garcia, Phys. Rev. B 74, 035212 (2006).

${ }^{4}$ Y. Yacoby, Phys. Rev. 169, 610 (1968).

${ }^{5}$ A. Srivastava, R. Srivastava, J. Wang, and J. Kono, Phys. Rev. Lett. 93, 157401 (2004).

${ }^{6}$ A.-P. Jauho and K. Johnsen, Phys. Rev. Lett. 76, 4576 (1996)

${ }^{7}$ K. Johnsen and A.-P. Jauho, Phys. Rev. B 57, 8860 (1998).

${ }^{8}$ K. Johnsen and A.-P. Jauho, Phys. Rev. Lett. 83, 1207 (1999).

${ }^{9}$ K. B. Nordstrom, K. Johnsen, S. J. Allen, A.-P. Jauho, B. Birnir, J. Kono, T. Noda, H. Akiyama, and H. Sakaki, Phys. Rev. Lett. 81, 457 (1998).

${ }^{10}$ S. Hughes and D. Citrin, Phys. Rev. B 60, 13272 (1999).

${ }^{11}$ S. Hughes and D. Citrin, Phys. Rev. B 59, R5288 (1999).

${ }^{12}$ C. J. Dent, B. N. Murdin, and I. Galbraith, Phys. Rev. B 67, 165312 (2003).

${ }^{13}$ S. Hughes, Phys. Rev. B 69, 205308 (2004).

${ }^{14}$ T. Y. Zhang and W. Zhao, Phys. Rev. B 73, 245337 (2006).

${ }^{15}$ H. T. Duc, T. Meier, and S. W. Koch, Phys. Rev. Lett. 95, 086606 (2005).

${ }^{16}$ T. Meier, G. von Plessen, P. Thomas, and S. Koch, Phys. Rev. Lett. 73, 902 (1994).

${ }^{17}$ T. Meier, G. von Plessen, P. Thomas, and S. Koch, Phys. Rev. B 51, 14490 (1995).

${ }^{18}$ T. Meier, F. Rossi, P. Thomas, and S. Koch, Phys. Rev. Lett. 75, 2558 (1995).

${ }^{19}$ S. H. Autler and C. H. Townes, Phys. Rev. 100, 703 (1955).

${ }^{20}$ T. Y. Zhang, W. Zhao, J. C. Cao, and G. Qasim, J. Appl. Phys. 98, 094311 (2005).

${ }^{21}$ I. Haque and M. R. Singh, J. Phys.: Condens. Matter 19, 156229 (2007).

${ }^{22}$ J. Cěrne, J. Kono, T. Inoshita, M. Sherwin, M. Sundaram, and A. C. Gossard, Appl. Phys. Lett. 70, 3543 (1997).

${ }^{23}$ S. G. Carter, V. Ciulin, M. S. Sherwin, M. Hanson, A. Huntington, L. A. Coldren, and A. C. Gossard, Appl. Phys. Lett. 84, 840 (2004). 